\title{
THE "MAXIMAL" TENSOR PRODUCT OF OPERATOR SPACES
}

\author{
by TIMUR OIKHBERG and GILLES PISIER*
}

(Received 12th February 1997)

\begin{abstract}
In analogy with the maximal tensor product of $C^{*}$-algebras, we define the "maximal" tensor product $E_{1} \otimes_{\mu} E_{2}$ of two operator spaces $E_{1}$ and $E_{2}$ and we show that it can be identified completely isometrically with the sum of the two Haagerup tensor products: $E_{1} \otimes_{h} E_{2}+E_{2} \otimes_{h} E_{1}$. We also study the extension to more than two factors. Let $E$ be an $n$-dimensional operator space. As an application, we show that the equality $E^{*} \otimes_{\mu} E=E^{*} \otimes_{\min } E$ holds isometrically iff $E=R_{n}$ or $E=C_{n}$ (the row or column $n$-dimensional Hilbert spaces). Moreover, we show that if an operator space $E$ is such that, for any operator space $F$, we have $F \otimes_{\min } E=F \otimes_{\mu} E$ isomorphically, then $E$ is completely isomorphic to either a row or a column Hilbert space.
\end{abstract}

1991 Mathematics subject classification: 47D15, 47D25, 46M05.

\section{Introduction}

In $C^{*}$-algebra theory, the minimal and maximal tensor products (denoted by $A_{1} \otimes_{\min } A_{2}$ and $A_{1} \otimes_{\max } A_{2}$ ) of two $C^{*}$-algebras $A_{1}, A_{2}$, play an important rôle, in connection with "nuclearity" (a $C^{*}$-algebra $A_{1}$ is nuclear if $A_{1} \otimes_{\min } A_{2}=A_{1} \otimes_{\max } A_{2}$ for any $A_{2}$ ). See [34] and [23] for more information and references on this. In the recently developed theory of operator spaces $[12-16,32,33,4,1-3]$, some specific new versions of the injective and projective tensor products (going back to Grothendieck for Banach spaces) have been introduced. The "injective" tensor product of two operator spaces $E_{1}, E_{2}$ coincides with the minimal (or spatial) tensor product and is denoted by $E_{1} \otimes_{\min } E_{2}$. Another tensor product of paramount importance for operator spaces is the Haagerup tensor product, denoted by $E_{1} \otimes_{h} E_{2}$ (cf. $[9,10,25,6,7]$ ). Assume given two completely isometric embeddings $E_{1} \subset A_{1}, E_{2} \subset A_{2}$. Then $E_{1} \otimes_{\min } E_{2}$ (resp. $E_{1} \otimes_{h} E_{2}$ ) can be identified with the closure of the algebraic tensor product $E_{1} \otimes E_{2}$ in $A_{1} \otimes_{\min } A_{2}$ (resp. in the "full" free product $C^{*}$-algebra $A_{1} * A_{2}$, see [8]). (The "projective" case apparently cannot be described in this fashion and will not be considered here.) It is therefore tempting to study the norm induced on $E_{1} \otimes E_{2}$ by $A_{1} \otimes_{\max } A_{2}$. When $A_{i}=B\left(H_{i}\right)(i=1,2)$ the resulting tensor product is studied in [19] and denoted by $E_{1} \otimes_{M} E_{2}$. See also [21] for other tensor products. In the present paper, we follow a different route: we work in the category of (a priori non self-adjoint) unital

* Partially supported by the N.S.F. 
operator algebras, and we use the maximal tensor product in the latter category (already considered in [24]), which extends the $C^{*}$-case.

The resulting tensor product, denoted by $E_{1} \otimes_{\mu} E_{2}$ is the subject of this paper. A brief description of it is as follows: we first introduce the canonical embedding of any operator space $E$ into an associated "universal" unital operator algebra, denoted by $O A(E)$, then we can define the tensor product $E_{1} \otimes_{\mu} E_{2}$ as the closure of $E_{1} \otimes E_{2}$ in $O A\left(E_{1}\right) \otimes_{\max } O A\left(E_{2}\right)$.

Our main result is Theorem 1 which shows that $E_{1} \otimes_{\mu} E_{2}$ coincides with a certain "symmetrization" of the Haagerup tensor product. We apply this (see Corollary 10 and Theorem 16) to find which spaces $E_{1}$ have the property that $E_{1} \otimes_{\mu} E_{2}=E_{1} \otimes_{\min } E_{2}$ for all operator spaces $E_{2}$. We give two proofs of the main result, in the bilinear case. The second one (in Section 2) is shorter, but we feel the first proof is more instructive, and easier to generalize to more general situations (cf. Remark 20). Besides, each proof seems to yield a different $n$-linear extension for $n>2$, not obtainable (as far as we can see) by the other argument (see the extension of Theorem 1, stated after its proof, and Theorem 19).

We refer the reader to the book [23] for the precise definitions of all the undefined terminology related to operator spaces and complete boundedness, and to $[26,34]$ for operator algebras in general. We recall only that an "operator space" is a closed subspace $E \subset B(H)$ of the $C^{*}$-algebra of all bounded operators on a Hilbert space $H$. We will use freely the notion of a completely bounded (in short c.b.) map $u: E_{1} \rightarrow E_{2}$ between two operator spaces, as defined e.g. in [23]. We denote by $\|u\|_{c b}$ the corresponding norm and by $c b\left(E_{1}, E_{2}\right)$ the Banach space of all c.b. maps from $E_{1}$ to $E_{2}$. We will denote by $A^{\prime}$ the commutant of a subset $A \subset B(H)$.

Let $E_{1}, \ldots, E_{n}$ be a family of operator spaces. Let $\sigma_{i}: E_{i} \rightarrow B(H)$ be complete contractions $(i=1,2, \ldots, n)$. We denote by $\sigma_{1} \cdot \ldots \cdot \sigma_{n}: E_{1} \otimes \cdots \otimes E_{n} \rightarrow B(H)$ the linear map taking $x_{1} \otimes \cdots \otimes x_{n}$ to the operator $\sigma_{1}\left(x_{1}\right) \sigma_{2}\left(x_{2}\right) \ldots \sigma_{n}\left(x_{n}\right)$.

We define the norm \|\|$_{\mu}$ on $E_{1} \otimes \cdots \otimes E_{n}$ as follows:

$$
\forall x \in E_{1} \otimes \cdots \otimes E_{n} \quad\|x\|_{\mu}=\sup \left\|\sigma_{1} \cdot \ldots \cdot \sigma_{n}(x)\right\|_{B(H)}
$$

where the supremum runs over all possible $H$ and all $n$-tuples $\left(\sigma_{i}\right)$ of complete contractions as above, with the restriction that we assume that for any $i \neq j$, the range of $\sigma_{i}$ commutes with the range of $\sigma_{j}$. We will denote by $\left(E_{1} \otimes E_{2} \cdots \otimes E_{n}\right)_{\mu}$ the completion of $E_{1} \otimes \cdots \otimes E_{n}$ for this norm. in the particular case $n=2$, we denote this simply by $E_{1} \otimes_{\mu} E_{2}$, so we have for any $x=\sum x_{i}^{1} \otimes x_{i}^{2} \in E_{1} \otimes E_{2}$

$$
\|x\|_{\mu}=\sup \left\{\left\|\sum \sigma_{1}\left(x_{i}^{1}\right) \sigma_{2}\left(x_{i}^{2}\right)\right\|_{B(H)}\right\}
$$

where the supremum runs over all possible pairs $\left(\sigma_{1}, \sigma_{2}\right)$ of complete contractions (into some common $B(H)$ ) with commuting ranges, i.e., such that $\sigma_{1}\left(x_{1}\right) \sigma_{2}\left(x_{2}\right)=\sigma_{2}\left(x_{2}\right) \sigma_{1}\left(x_{1}\right)$ for all $x_{1} \in E_{1}, x_{2} \in E_{2}$.

The space $\left(E_{1} \otimes \cdots \otimes E_{n}\right)_{\mu}$ can obviously be equipped with an operator space 
structure associated to the embedding

$$
J:\left(E_{1} \otimes \cdots \otimes E_{n}\right)_{\mu} \rightarrow \bigoplus_{\sigma} B\left(H_{\sigma}\right) \subset B\left(\bigoplus_{\sigma} H_{\sigma}\right)
$$

where the direct sum runs over all $n$-tuples $\sigma=\left(\sigma_{1}, \ldots, \sigma_{n}\right)$ with $\sigma_{i}: E_{i} \rightarrow B\left(H_{\sigma}\right)$ such that $\left\|\sigma_{i}\right\|_{c b} \leq 1$ and the $\sigma_{i}$ 's have commuting ranges. Here as well as throughout this paper, we observe that we can always restrict ourselves in the above direct sum to the case when the cardinal of $H_{\sigma}$ is majorized by a suitably fixed cardinal, thus eliminating set theoretic objections.

We note in passing that if we define $\hat{\sigma}_{i}: E_{i} \rightarrow \oplus_{\sigma} B\left(H_{\sigma}\right) \subset B\left(\oplus_{\sigma} H_{\sigma}\right)$ by $\hat{\sigma}_{i}(x)=\oplus_{\sigma} \sigma_{i}(x)$, then we have $J(x)=\hat{\sigma}_{1} \ldots \hat{\sigma}_{n}$ and the maps $\hat{\sigma}_{i}$ have commuting ranges.

Thus we can now unambiguously refer to $\left(E_{1} \otimes \cdots \otimes E_{n}\right)_{\mu}$, and in particular to $E_{1} \otimes_{\mu} E_{2}$ as operator spaces.

We will give more background on operator spaces and c.b. maps below. For the moment, we merely define a "complete metric surjection": by this we mean a surjective mapping $Q: E_{1} \rightarrow E_{2}$ between two operator spaces, which induces a complete isometry from $E_{1} / \operatorname{ker}(Q)$ onto $E_{2}$.

To state our main result, we also need the notion of $\ell_{1}$-direct sum of two operator spaces $E_{1}, E_{2}$ : this is an operator space denoted by $E_{1} \oplus_{1} E_{2}$. The norm on the latter space is as in the usual $\ell_{1}$-direct sum, i.e., we have

$$
\left\|\left(e_{1}, e_{2}\right)\right\|_{E_{1} \oplus_{1} E_{2}}=\left\|e_{1}\right\|_{E_{1}}+\left\|e_{2}\right\|_{E_{2}}
$$

but the operator space structure is such that for any pair $u_{1}: E_{1} \rightarrow B(H)$, $u_{2}: E_{2} \rightarrow B(H)$ of complete contractions, the mapping $\left(e_{1}, \dot{e}_{2}\right) \rightarrow u_{1}\left(e_{1}\right)+u_{2}\left(e_{2}\right)$ is a complete contraction from $E_{1} \oplus_{1} E_{2}$ to $B(H)$.

The simplest way to realize this operator space $E_{1} \oplus_{1} E_{2}$ as a subspace of $B(\mathcal{H})$ for some $\mathcal{H}$ is to consider the collection $I$ of all pairs $p=\left(u_{1}, u_{2}\right)$ as above with $H=H_{p}$ (say) and to define the embedding

$$
J: E_{1} \oplus_{1} E_{2} \rightarrow B\left(\bigoplus_{p \in I} H_{p}\right)
$$

defined by $J\left(e_{1}, e_{2}\right)=\oplus_{\left(u_{1}, u_{2}\right) \in I}\left[u_{1}\left(e_{1}\right)+u_{2}\left(e_{2}\right)\right]$. Then, we may as well define the operator space structure of $E_{1} \oplus_{1} E_{2}$ as the one induced by the isometric embedding $J$. In other words $E_{1} \oplus_{1} E_{2}$ can be viewed as the "maximal" direct sum for operator spaces, in accordance with the general theme of this paper.

We will denote by $E_{1} \otimes E_{2}$ the linear tensor product of two vector spaces and by $v \rightarrow{ }^{\prime} v$ the transposition map, i.e., for any $v=\sum x_{i} \otimes y_{i}$ in $E_{1} \otimes E_{2}$, we set ${ }^{\prime} v=\sum y_{i} \otimes x_{i}$. The identity map on a space $E$ will be denoted by $I d_{E}$.

We will denote by $E_{1} \otimes_{h} E_{2}$ the Haagerup tensor product of two operator spaces for which we refer to $[9,10,25]$. 
Convention. We reserve the term "morphism" for a unital completely contractive homomorphism $u: A \rightarrow B$ between two unital operator algebras.

\section{Main results}

Our main result is the following one.

Theorem 1 (bilinear case). Let $E_{1}, E_{2}$ be two operator spaces. Consider the mapping

$$
Q:\left(E_{1} \otimes_{h} E_{2}\right) \oplus_{1}\left(E_{2} \otimes_{h} E_{1}\right) \rightarrow E_{1} \otimes_{\mu} E_{2}
$$

defined on the direct sum of the linear tensor products by $Q(u \oplus v)=u+{ }^{t} v$. Then $Q$ extends to a complete metric surjection from $\left(E_{1} \otimes_{h} E_{2}\right) \oplus_{1}\left(E_{2} \otimes_{h} E_{1}\right)$ onto $E_{1} \otimes_{\mu} E_{2}$. In particular, for any $u$ in $E_{1} \otimes E_{2}$, we have: $\|u\|_{\mu}<1$ iff there are $v, w$ in $E_{1} \otimes E_{2}$ such that $u=v+w$ and $\|v\|_{E_{1} \otimes_{h} E_{2}}+\|\|^{t} w \|_{E_{2} \otimes_{h} E_{1}}<1$.

In the terminology of [28], the preceding statement means that $E_{1} \otimes_{\mu} E_{2}$ is completely isometric to the "sum" (in the style of interpolation theory, see [28]) $E_{1} \otimes_{h} E_{2}+E_{2} \otimes_{h} E_{1}$ (in analogy with $R+C$ ).

Remark 2. We first recall a simple consequence of the Cauchy-Schwarz inequality: for any $a_{1}, \ldots, a_{n}, b_{1}, \ldots, b_{n}$ in a $C^{*}$-algebra $A$, we have $\left\|\sum a_{i} b_{i}\right\| \leq\left\|\sum a_{i} a_{i}^{*}\right\|^{1 / 2}\left\|\sum b_{i}^{*} b_{i}\right\|^{1 / 2}$. Hence if $a_{i} b_{i}=b_{i} a_{i}$ we also have $\left\|\sum a_{i} b_{i}\right\| \leq\left\|\sum a_{i}^{*} a_{i}\right\|^{1 / 2}\left\|\sum b_{i} b_{i}^{*}\right\|^{1 / 2}$. From these it is easy to deduce that the above map $Q$ is completely contractive.

The main idea of the proof of Theorem 1 is to use the universal unital operator algebras of operator spaces as initiated in [30], to relate their free product with their "maximal" tensor product, and to use the appearance of the Haagerup tensor product inside the free product.

Let $E$ be an operator space. Let $T(E)=\mathbf{C} \oplus E \oplus(E \otimes E) \oplus \cdots$ be its tensor algebra, so that any $x$ in $T(E)$ is a sum $x=\sum x_{n}$ with $x_{n} \in E \otimes \cdots \otimes E$ ( $n$ times) with $x_{n}=0$ for all $n$ sufficiently large. For each linear $\sigma: E \rightarrow B(H)$ we denote by $T(\sigma): T(E) \rightarrow B(H)$ the unique unital homomorphism extending $\sigma$.

Let $C$ be the collection of all $\sigma: E \rightarrow B\left(H_{a}\right)$ with $\|\sigma\|_{c b} \leq 1$. We define an embedding

$$
J: T(E) \rightarrow B\left(\bigoplus_{\sigma \in C} H_{\sigma}\right)
$$

by setting

$$
J(x)=\bigoplus_{\sigma \in C} T(\sigma)(x)
$$


Then $J$ is a unital homomorphism. We denote by $O A(E)$ the unital operator algebra obtained by completing $J(T(E)$ ). We will always view $T(E)$ as a subset of $O A(E)$, so we identify $x$ and $J(x)$ when $x \in T(E)$. Observe that the natural inclusion

$$
E \rightarrow O A(E)
$$

is obviously a complete isometry. More generally, the natural inclusion of $E \otimes \cdots \otimes E$ ( $n$ times) into $O A(E)$ defines a completely isometric embedding of $E \otimes_{h} \cdots \otimes_{h} E$ into $O A(E)$. (This follows from a trick due to Varopoulos, and used by Blecher in [1], see [30] for details.)

The algebra $O A(E)$ is characterized by the following universal property: for any $\sigma: E \rightarrow B(H)$ with $\|\sigma\|_{c b} \leq 1$, there is a unique morphism $\hat{\sigma}: O A(E) \rightarrow B(H)$ extending $\sigma$ (here we view $E$ as embedded into $O A(E)$ in the natural way). See [26] for the selfadjoint analogue.

We now turn to the maximal tensor product in the category of unital operator algebras. This is defined in [24], so we only briefly recall the definition: Let $A_{1}, A_{2}$ be two unital operator algebras. For any pair $\pi=\left(\pi_{1}, \pi_{2}\right)$ of morphisms $\pi_{i}: A_{i} \rightarrow B\left(H_{\pi}\right)$ with commuting ranges, we denote by $\pi_{1} \cdot \pi_{2}$ the morphism from $A_{1} \otimes A_{2}$ to $B\left(H_{\pi}\right)$ which takes $a_{1} \otimes a_{2}$ to $\pi_{1}\left(a_{1}\right) \pi_{2}\left(a_{2}\right)$. Then we consider the embedding

$$
J: A_{1} \otimes A_{2} \rightarrow B\left(\bigoplus_{\pi} H_{\pi}\right)
$$

defined by $J(x)=\oplus_{\pi} \pi_{1} \cdot \pi_{2}(x)$. We define

$$
\|x\|_{\max }=\sup _{\pi}\left\|\pi_{1} \cdot \pi_{2}(x)\right\|
$$

and we denote by $A_{1} \otimes_{\max } A_{2}$ the completion of $A_{1} \otimes A_{2}$ for this norm. We will consider $A_{1} \otimes_{\max } A_{2}$ as a unital operator algebra, using the isometric embedding $J$ just defined.

Lemma 3. The natural inclusion of $E_{1} \otimes_{\mu} E_{2}$ into $O A\left(E_{1}\right) \otimes_{\max } O A\left(E_{2}\right)$ is a completely isometric embedding.

We now turn to the free product in the category of unital operator algebras. Let $A_{1}, A_{2}$ be two such algebras and let $\mathcal{F}$ be their algebraic free product as unital algebras (i.e., we identify the units and "amalgamate over C"). For any pair $u=\left(u_{1}, u_{2}\right)$ of morphisms, as follows $u_{i}: A_{i} \rightarrow B\left(H_{u}\right)(i=1,2)$, we denote by $u_{1} * u_{2}: \mathcal{F} \rightarrow B\left(H_{u}\right)$ the unital homomorphism extending $u_{1}, u_{2}$ to the free product. Then we consider the embedding

$$
J: \mathcal{F} \rightarrow \bigoplus_{u} B\left(H_{u}\right)
$$


defined by $J(x)=\oplus_{u}\left[u_{1} * u_{2}(x)\right]$, for all $x$ in $\mathcal{F}$. Note that $J$ is a unital homomorphism.

We define the free product $A_{1} * A_{2}$ (in the category of unital operator algebras) as the closure of $J(\mathcal{F})$. Actually, we will identify $\mathcal{F}$ with $J(\mathcal{F})$ and consider that $A_{1} * A_{2}$ is the completion of $\mathcal{F}$ relative to the norm induced by $J$. Moreover, we will consider $A_{1} * A_{2}$ as a unital operator algebra equipped with the operator space structure induced by $J$.

It is easy to see that $A_{1} * A_{2}$ is characterized by the (universal) property that for any pair of morphisms $u_{i}: A_{i} \rightarrow B(H)(i=1,2)$ there is a unique morphism from $A_{1} * A_{2}$ to $B(H)$ which extends both $u_{1}$ and $u_{2}$.

We will use several elementary facts which essentially all follow from the universal properties of the objects we have introduced.

Lemma 4. $O A\left(E_{1}\right) * O A\left(E_{2}\right) \simeq O A\left(E_{1} \oplus_{1} E_{2}\right)$ completely isometrically.

Remark. The "functor" $E \rightarrow O A(E)$ is both injective and projective: i.e., if $E_{2} \subset E_{1}$ is a closed subspace then the associated morphisms $j: O A\left(E_{2}\right) \rightarrow O A\left(E_{1}\right)$ and $q$ : $O A\left(E_{1}\right) \rightarrow O A\left(E_{1} / E_{2}\right)$ are respectively a complete isometry and a complete metric surjection. The injectivity is easy. To check the projectivity, let $B=O A\left(E_{1}\right) / \operatorname{ker}(q)$ and let $\hat{q}: B \rightarrow O A\left(E_{1} / E_{2}\right)$ be the completely contractive morphism canonically associated to $q$. Note that we have a complete contraction $E_{1} \rightarrow O A\left(E_{1}\right) \rightarrow B$ which vanishes on $E_{2}$, whence a complete contraction $E_{1} / E_{2} \rightarrow B$, which extends to a completely contractive morphism $O A\left(E_{1} / E_{2}\right) \rightarrow B$. The latter morphism is inverse to $\hat{q}$, hence $\hat{q}$ is a complete isometry.

We will use the following fact which was observed by Blecher and Paulsen ([5, 4.41).

Lemma 5. Let $A_{1}, A_{2}$ be two unital operator algebras. Then the natural morphism $Q: A_{1} * A_{2} \rightarrow A_{1} \otimes_{\max } A_{2}$ is a complete metric surjection. More precisely, the restriction of $Q$ to the algebraic free product $\mathcal{F}$ defines a complete isometry between $\mathcal{F} / \operatorname{ker}\left(Q_{\mid \mathcal{F}}\right)$ and $A_{1} \otimes A_{2} \subset A_{1} \otimes_{\max } A_{2}$.

The next lemma (already used in [1]) is elementary.

Lemma 6. Consider an element $x=x_{0}+x_{1}+\cdots+x_{n}+\cdots$ in $T(E)$, with $x_{n} \in E \otimes \cdots \otimes E$ ( $n$ times). Then the mapping $x \rightarrow x_{n}$ defines a completely contractive projection on $O A(E)$.

Proof. Let $m$ denote the normalized Haar measure on the unidimensional torus $\mathbf{T}$. For $z$ in $\mathrm{T}$, let $x(z)=\sum_{n \geq 0} z^{n} x_{n}$. By definition of $O A(E)$, we clearly have $\|x(z)\|=\|x\|$, hence 


$$
\left\|x_{n}\right\|=\left\|\int z^{-n} x(z) m(d z)\right\| \leq\|x\| .
$$

This shows that $x \rightarrow x_{n}$ is a contractive linear projection. The argument for complete contractivity is analogous and left to the reader.

The next result, which plays an important rôle in the sequel, might be of independent interest.

Lemma 7. Let $E_{1}, E_{2}, F_{1}, F_{2}$ be four operator spaces. Let $X=\left(E_{1} \oplus_{1} E_{2}\right) \otimes_{h}\left(F_{1} \oplus_{1} F_{2}\right)$. With the obvious identifications, we may view $E_{1} \otimes F_{2}+E_{2} \otimes F_{1}$ as a linear subspace of $X$. Let $S$ be its closure in $X$. Then we have

$$
S \simeq\left(E_{1} \otimes_{h} F_{2}\right) \oplus_{1}\left(E_{2} \otimes_{h} F_{1}\right)
$$

completely isometrically. Moreover, the natural (coordinatewise) projection $P: X \rightarrow S$ is completely contractive.

Proof. Obviously we have completely contractive natural inclusions $E_{1} \otimes_{h} F_{2} \rightarrow X$ and $E_{2} \otimes_{h} F_{1} \rightarrow X$, whence a natural inclusion $\left(E_{1} \otimes_{h} F_{2}\right) \oplus_{1}\left(E_{2} \otimes_{h} F_{1}\right) \rightarrow X$. To show that this is completely isometric it clearly suffices to show that $S$ has the "universal" property characteristic of the $\oplus_{1}$-direct sum. Equivalently, it suffices to show that every completely contractive mapping $\sigma:\left(E_{1} \otimes_{h} F_{2}\right) \oplus_{1}\left(E_{2} \otimes_{h} F_{1}\right) \rightarrow B(H)$ defines a completely contractive mapping from $S$ to $B(H)$ (then we may apply this when $\sigma$ is a completely isometric embedding). So let $\sigma$ be such a map. Clearly, we can assume that $\sigma(x \oplus y)=u(x)+v(y) \quad$ with $u: E_{1} \otimes_{h} F_{2} \rightarrow B(H), \quad v: E_{2} \otimes_{h} F_{1} \rightarrow B(H)$ such that $\|u\|_{c b} \leq 1,\|v\|_{c b} \leq 1$. By the factorization of c.b.-bilinear maps ([9, 25]) we can further write $u\left(x_{1} \otimes x_{2}\right)=u_{1}\left(x_{1}\right) v_{2}\left(x_{2}\right)$ and $v\left(y_{2} \otimes y_{1}\right)=u_{2}\left(y_{2}\right) v_{1}\left(y_{1}\right)$ where $u_{i}: E_{i} \rightarrow B(H)$ and $v_{i}: F_{i} \rightarrow B(H)$ are all completely contractive. Let us then define $\alpha: E_{1} \oplus_{1} E_{2} \rightarrow B(H)$ and $\beta: F_{1} \oplus_{1} F_{2} \rightarrow B(H)$ by $\alpha\left(x_{1} \oplus x_{2}\right)=u_{1}\left(x_{1}\right)+u_{2}\left(x_{2}\right)$ and $\beta\left(x_{1} \oplus x_{2}\right)=v_{1}\left(x_{1}\right)+v_{2}\left(x_{2}\right)$. By definition of $\oplus_{1}$, these maps are still complete contractions. Moreover, we have for any $z$ in $S$, say $z=x+y$ with $x \in E_{1} \otimes F_{2}, y \in E_{2} \otimes F_{1}$

$$
\alpha \cdot \beta(z)=u(x)+v(y)=\sigma(z) .
$$

hence we conclude that $\sigma$ admits an extension $\tilde{\sigma}$ (namely $\tilde{\sigma}=\alpha \cdot \beta$ ) defined on the whole of $X$ with

$$
\|\tilde{\sigma}\|_{c b(X, B(H))} \leq\|\alpha\|_{c b}\|\beta\|_{c b} \leq 1,
$$

a fortiori $\|\sigma\|_{c b(S, B(H))} \leq 1$. This established the first part. To check that $P$ is completely contractive, just observe that if we denote by $Q_{i}$ (resp. $R_{i}$ ) the $i$-th canonical projection on $E_{1} \oplus_{1} E_{2}$ (resp. $\left.F_{1} \oplus_{1} F_{2}\right)$, then $P$ is equal to the average of $\left(\varepsilon_{1} Q_{1}+\varepsilon_{2} Q_{2}\right) \otimes$ $\left(\varepsilon_{2} R_{1}+\varepsilon_{1} R_{2}\right)$ over all the choices of signs $\left(\varepsilon_{i}\right)$. 
Lemma 8. Let $A_{1}, \ldots, A_{n}$ be unital operator algebras. Then the natural mapping from $A_{1} \otimes A_{2} \cdots \otimes A_{n}$ to $A_{1} * A_{2} * \cdots * A_{n}$ defines a completely isometric embedding of $A_{1} \otimes_{h} A_{2} \cdots \otimes_{h} A_{n}$ into $A_{1} * A_{2} * \cdots * A_{n}$.

Proof. In essence, this is proved in [8], but only for the non-unital free product. The unital case is done in detail in [29] so we skip it.

Proof of Theorem 1. Consider $u$ in $E_{1} \otimes E_{2}$ with $\|u\|_{\mu}<1$. Let $\mathcal{F}$ be, as before, the algebraic free product of $O A\left(E_{1}\right)$ and $O A\left(E_{2}\right)$. By Lemma 5, we have $\|u\|_{O A\left(E_{1}\right) \otimes_{\max } O A\left(E_{2}\right)}<1$, hence by Lemma 4 , there is an element $\hat{u}$ in $\mathcal{F}$ with $\|\hat{u}\|<1$ such that $Q(\hat{u})=u$. By Lemma 3 we may write as well $\|\hat{u}\|_{O A\left(E_{1} \oplus_{1} E_{2}\right)}<1$. Let us write $\hat{u}=\hat{u}_{0}+\hat{u}_{1}+\hat{u}_{2}+\cdots$ where $\hat{u}_{d} \in\left(E_{1} \oplus_{1} E_{2}\right) \otimes_{h} \cdots \otimes_{h}\left(E_{1} \oplus_{1} E_{2}\right)\left(d\right.$ times). By Lemma 6 we have $\left\|\hat{u}_{d}\right\|<1$. Let $z_{1}, z_{2}$ be complex numbers with $\left|z_{i}\right| \leq 1$. There is a unique morphism $\pi_{z_{i}}: O A\left(E_{i}\right) \rightarrow O A\left(E_{i}\right)$ extending $z_{i} I d_{E_{i}}$. We will use the morphisms

$$
\pi_{z 1} \otimes \pi_{z_{2}} \text { acting on } O A\left(E_{1}\right) \otimes_{\max } O A\left(E_{2}\right)
$$

and

$$
\pi_{z_{1}} * \pi_{z_{2}} \text { acting on } O A\left(E_{1}\right) * O A\left(E_{2}\right)
$$

Note that we trivially have the following relation:

$$
\left[\pi_{z_{1}} \otimes \pi_{z_{2}}\right] \circ Q=Q \circ\left[\pi_{z_{1}} * \pi_{z_{2}}\right] .
$$

It follows that $z_{1} z_{2} u=Q\left[\pi_{z_{1}} * \pi_{z_{2}}(\hat{u})\right]$. Hence identifying the coefficient of $z_{1} z_{2}$ on the right hand side we obtain $u=Q[\tilde{u}]$ where $\tilde{u}$ is in the subspace $S=\operatorname{span}\left[E_{1} \otimes E_{2}+E_{2} \otimes E_{1}\right] \subset\left(E_{1} \oplus_{1} E_{2}\right) \otimes_{h}\left(E_{1} \oplus_{1} E_{2}\right)$ considered in Lemma 7 , and where we view $\left(E_{1} \oplus_{1} E_{2}\right) \otimes_{h}\left(E_{1} \oplus_{1} E_{2}\right)$ as the subspace of $O A\left(E_{1} \oplus_{1} E_{2}\right)$ formed of all terms of degree 2 , according to Lemma 8 . Hence by Lemma 7 , we conclude that $\tilde{u}$ can be written as $v+w$ with $v \in E_{1} \otimes E_{2}$ and $w \in E_{2} \otimes E_{1}$ such that $\|v\|_{E_{1} \otimes_{h} E_{2}}+\|w\|_{E_{2} \otimes_{h} E_{1}}<1$. This shows that $\forall u \in E_{1} \otimes E_{2}$ with $\|u\|_{\mu}<1$ there are $v, w$ as above with $u=v+{ }^{t} w$.

Thus the natural mapping is a metric surjection from $E_{1} \otimes_{h} E_{2} \oplus_{1} E_{2} \otimes_{h} E_{1}$ onto $E_{1} \otimes_{\mu} E_{2}$. To show that this is a complete surjection, one simply repeats the argument with $M_{n}\left(E_{1} \otimes_{\mu} E_{2}\right)$ instead of $E_{1} \otimes_{\mu} E_{2}$. We leave the easy details to the reader.

Using the same techniques, one can prove the following isomorphic generalization of Theorem 1 for more than two spaces:

Theorem 1 (general case). Let $E_{1}, \ldots, E_{n}$ be an $n$-tuple of operator spaces. Let $L$ be the $\ell_{1}$-direct sum of the family $\left(E_{\sigma(1)} \otimes_{h} \ldots \otimes_{h} E_{\sigma(n)}\right)_{\sigma}$ indexed by all permutations $\sigma$ of $\{1,2, \ldots, n\}$. Let $Q$ be the natural completely contractive mapping from $L$ to 
$\left(E_{1} \otimes \cdots \otimes E_{n}\right)_{\mu}$, and let $\Phi: L / \operatorname{ker}(Q) \rightarrow\left(E_{1} \otimes \cdots \otimes E_{n}\right)_{\mu}$ be the canonically associated map. Then $\Phi$ is a complete isomorphism satisfying $\|\Phi\|_{c b} \leq 1$ and $\left\|\Phi^{-1}\right\|_{c b} \leq(n-1)$ !.

Proof. We will use the following fact. Let $E_{i j}$ be a family of operator spaces. Let

$$
\begin{aligned}
& X=\left(E_{11} \oplus_{1} \ldots \oplus_{1} E_{1 n}\right) \otimes_{h} \ldots \otimes_{h}\left(E_{k 1} \oplus_{1} \ldots \oplus_{1} E_{k n}\right) ; \\
& Y=\left(E_{11} \otimes_{h} \ldots \otimes_{h} E_{k 1}\right) \oplus_{1} \ldots \oplus_{1}\left(E_{1 n} \otimes_{h} \ldots \otimes_{h} E_{k n}\right) ; \\
& S=\left(E_{11} \otimes_{h} \ldots \otimes_{h} E_{k 1}\right) \oplus \ldots \oplus\left(E_{1 n} \otimes_{h} \ldots \otimes_{h} E_{k n}\right) \hookrightarrow X,
\end{aligned}
$$

then $Y \simeq S$ completely isometrically (here $S$ is equipped with the operator space structure induced by $X$ ). Moreover, the natural (coordinatewise) projection $P: X \rightarrow S$ is completely contractive. This follows from Lemma 7 by iteration. We skip the details.

Let us now prove the preceding statement. Let $E=E_{1} \oplus_{1} \ldots \oplus_{1} E_{n}$. Let $G_{n}$ be the set of all permutations of $\{1,2, \ldots, n\}$. Let $\mathcal{X}=E \otimes_{h} \ldots \otimes_{h} E$ ( $n$ times) and let $\Lambda \subset \mathcal{X}$ be the subspace defined by $\Lambda=\sum_{\sigma \in G_{n}} E_{\sigma(1)} \otimes \ldots \otimes E_{\sigma(n)}$. For any subset $A \subset G_{n}$, we set $\Lambda(A)=\sum_{\sigma \in A} E_{\sigma(1)} \otimes \ldots \otimes E_{\sigma(n)}$. We equip $\Lambda$ and $\Lambda(A)$ with the operator space structure induced by $\mathcal{X}$. Let us say that $A$ is admissible if for each $i$ the set $\{\sigma(i) \mid \sigma \in A\}$ is the whole of $\{1,2, \ldots, n\}$.

By the same argument as above for $n=2$, the natural product map $Q: \Lambda \rightarrow\left(E_{1} \otimes \cdots \otimes E_{n}\right)_{\mu}$ is a complete metric surjection. We clearly have a natural completely contractive map $\psi: L \rightarrow \Lambda$. To conclude, it suffices to prove that $\psi$ is a complete isomorphism with $\left\|\psi^{-1}\right\|_{c b} \leq(n-1)$ !. To prove this we use a partition of $G_{n}$ into $(n-1)$ ! admissible subsets, each with $n$ elements (for instance the left cosets associated with the subgroup formed of all the $n$ cyclic permutations). Indeed, by the preceding fact, for any admissible $A$, the restriction of $\psi^{-1}$ to $\Lambda(A)$ is a complete isometry, and the natural projection from $\Lambda$ to $\Lambda(A)$ is completely contractive. This implies $\left\|\psi^{-1}\right\|_{c b} \leq(n-1)$ !

Remark. We do not believe that the isometric analogue of Theorem 1 holds true for $n>2$. While we do not have an explicit example, at least we have checked that the map $\psi$ appearing above is not completely isometric in general.

Remark. If $X, Y$ are Banach spaces, and if $v \in Y \otimes X$, let us denote by $\gamma_{2}(v)$ the norm of factorization through Hilbert space of the linear map $\tilde{v}: Y^{*} \rightarrow X$ associated to $v$. This is a classical notion in Banach space theory (c.f. e.g. [27, p. 21]). Note that Theorem 1 obviously implies that for any $v$ in $E_{1} \otimes E_{2}\left(E_{1}, E_{2}\right.$ being arbitrary operator spaces), we have

$$
\gamma_{2}(v) \leq\|v\|_{\mu} .
$$

Remark. Note that

$\left(E_{1} \otimes_{\mu} E_{2}\right) \otimes_{\mu} E_{3}=E_{1} \otimes_{h} E_{2} \otimes_{h} E_{3}+E_{3} \otimes_{h} E_{1} \otimes_{h} E_{2}+E_{2} \otimes_{h} E_{1} \otimes_{h} E_{3}+E_{3} \otimes_{h} E_{2} \otimes_{h} E_{1}$, 
but the above expression does not necessarily coincide with $\left(E_{1} \otimes E_{2} \otimes E_{3}\right)_{\mu}$, and moreover the $\mu$-tensor product is not associative, in sharp contrast with the Haagerup one (or with the maximal tensor product for unital operator algebras). In particular, in general the natural mapping from $E_{1} \otimes_{\mu}\left(E_{2} \otimes_{\mu} E_{3}\right)$ into $\left(E_{1} \otimes_{\mu} E_{2}\right) \otimes_{\mu} E_{3}$ is unbounded (and actually only makes sense on the linear tensor products). All this follows from the counterexample below, kindly communicated to us by C. Le Merdy. Let $X$ be a Banach space and let $K$ be the algebra of all compact operators on $\ell_{2}$. Take $E_{1}=C, E_{2}=R$, and $E_{3}=\min (X)$ in the sense of [4]. Assume that we have a bounded map

$$
\left(E_{1} \otimes E_{2} \otimes E_{3}\right)_{\mu} \rightarrow\left(E_{1} \otimes_{\mu} E_{2}\right) \otimes_{\mu} E_{3} .
$$

Then a fortiori we have a bounded map $E_{1} \otimes_{\mu}\left(E_{2} \otimes_{\mu} E_{3}\right) \rightarrow\left(E_{1} \otimes_{\mu} E_{2}\right) \otimes_{\mu} E_{3}$, and consequently a bounded map $E_{1} \otimes_{h} E_{3} \otimes_{h} E_{2} \rightarrow\left(E_{1} \otimes_{\mu} E_{2}\right) \otimes_{\mu} E_{3}$. But then $C \otimes_{h} E_{3} \otimes_{h} R=$ $K \otimes_{\min } E_{3}$ completely isometrically (see $[4,14]$ ), hence it is isometric to the (Banach space theoretic) injective tensor product $K \ddot{\otimes} X$. Moreover, since $R \otimes_{h} C$ is isometric to $K^{*}$, by Theorem 1 we have $C \otimes_{\mu} R=K$ isometrically.

Thus, we would have a bounded map from $K \ddot{\otimes} X$ to $\left(C \otimes_{\mu} R\right) \otimes_{\mu} E_{3}$, and this would imply by (3), that for some constant $C$, for all $v$ in $K \ddot{\otimes} X$, we would have $\gamma_{2}(v) \leq C\|v\|_{\mathrm{v}}$. However, it is well known that this fails at least for some Banach space $X$ (take for example $X=\ell_{1}$ and $v \sum_{1}^{n} e_{i i} \otimes e_{i}$, so that $v$ represents an isomorphic embedding of $\ell_{n}^{\infty}$ into $K$, then $\|v\|_{v}=1$ and $\gamma_{2}(v)=\sqrt{n}$, c.f. [27, p. 48] for more on this question).

We now give several consequences and reinterpret Theorem 1, in terms of factorization.

The following notation will be convenient. Let $X$ be an operator space. We will say that a linear map $u: E_{1} \rightarrow E_{2}$ between operator spaces factors through $X$ if there are maps $w: E_{1} \rightarrow X$ and $v: X \rightarrow E_{2}$ such that $u=v w$. We will denote by $\Gamma_{X}\left(E_{1}, E_{2}\right)$ the class of all such mappings and moreover we let

$$
\gamma_{X}(u)=\inf \left\{\|v\|_{c b}\|w\|_{c b}\right\}
$$

where the infimum runs over all possible such factorizations. Let us denote by $\mathcal{K}$ the $C^{*}$-algebra of all compact operators on $\ell_{2}$, with its natural "basis" $\left(e_{i j}\right)$.

The preceding notation applies in particular when $X=\mathcal{K}$ and gives us the space $\Gamma_{\mathcal{K}}\left(E_{1}, E_{2}\right)$. In the case $X=\mathcal{K}$, it is easy to check that $\gamma_{\mathcal{K}}$ is a norm with which $\Gamma_{\mathcal{K}}\left(E_{1}, E_{2}\right)$ becomes a Banach space.

We wish to relate the possible factorizations of a map through $\mathcal{K}$ with its possible factorizations through two specific subspaces of $\mathcal{K}$, namely the row and column Hilbert spaces defined by

$$
\begin{aligned}
& R=\overline{\operatorname{span}}\left(e_{1 j} \mid j=1,2, \ldots\right) \\
& C=\overline{\operatorname{span}}\left(e_{i \mid} \mid i=1,2, \ldots\right) .
\end{aligned}
$$

Clearly these subspaces of $\mathcal{K}$ admit a natural completely contractive projection onto 
them (namely $x \rightarrow e_{11} x$ is a projection onto $R$, and $x \rightarrow x e_{11}$ one onto $C$ ). Therefore we have $\gamma_{\mathcal{K}}\left(I d_{R}\right)=1$ and $\gamma_{\mathcal{K}}\left(I d_{C}\right)=1$. A fortiori any linear map $u: E_{1} \rightarrow E_{2}$ which factors either through $R$ or through $C$ factors through $\mathcal{K}$ and we have

$$
\gamma_{K}(u) \leq \gamma_{R}(u) \text { and } \gamma_{K}(u) \leq \gamma_{c}(u)
$$

A fortiori, if $u=v+w$ for some $v: E_{1} \rightarrow E_{2}$ and $w: E_{1} \rightarrow E_{2}$, we have

$$
\gamma_{\mathcal{K}}(u) \leq \gamma_{R}(v)+\gamma_{C}(w)
$$

Note that if $v$ and $w$ are of finite rank, then with the obvious identifications, we have

$$
\gamma_{R}(v)=\|v\|_{E_{1}^{*} \otimes_{h} E_{2}} \quad \text { and } \quad \gamma_{C}(w)=\|\|_{E_{2} \otimes_{h} E_{1}} .
$$

Thus, from Theorem 1 we deduce:

Corollary 9. Let $E_{1}, E_{2}$ be operator spaces. Consider $u$ in $E_{1}^{*} \otimes E_{2}$ and let $\bar{u}: E_{1} \rightarrow E_{2}$ be the associated finite rank operator. Then we have

$$
\gamma_{\mathcal{K}}(\tilde{u}) \leq\|u\|_{\mu}
$$

Corollary 10. Let $E$ be an n-dimensional operator space. Let $i_{E} \in E^{*} \otimes E$ be associated to the identity of $E$ and let

$$
\mu(E)=\left\|i_{E}\right\|_{\mu}
$$

Then

$$
\max \left\{\gamma_{\mathcal{K}}\left(I d_{E}\right), \gamma_{\mathcal{K}}\left(I d_{E^{*}}\right)\right\} \leq \mu(E)
$$

Moreover $\mu(E)=1$ iff either $E=R_{n}$ or $E=C_{n}$ (completely isometrically).

Proof. Note that (5) clearly follows from (4). Assume that $\mu(E)=1$. Then by Theorem 1 (and an obvious compactness argument) we have a decomposition $I d_{E}=u_{1}+u_{2}$ with

$$
\gamma_{R}\left(u_{1}\right)+\gamma_{c}\left(u_{2}\right)=1
$$

In particular, this implies that $\gamma_{2}\left(I d_{E}\right)=1$, (where $\gamma_{2}($.$) denotes the norm of$ factorization through Hilbert space, see e.g. [27, Chapter 2] for more background) whence that $E$ is isometric to $\ell_{2}^{n}(n=\operatorname{dim} E)$. Moreover, for any $e$ in the unit sphere of $E$ we have 


$$
1=\|e\| \leq\left\|u_{1}(e)\right\|+\left\|u_{2}(e)\right\| \leq\left\|u_{1}\right\|+\left\|u_{2}\right\| \leq \gamma_{R}\left(u_{1}\right)+\gamma_{C}\left(u_{2}\right) \leq 1 .
$$

Therefore we must have

$$
\left\|u_{1}(e)\right\|=\left\|u_{1}\right\|=\gamma_{R}\left(u_{1}\right) \text { and }\left\|u_{2}(e)\right\|=\left\|u_{2}\right\|=\gamma_{C}\left(u_{2}\right) .
$$

Let $\alpha_{i}=\left\|u_{i}\right\|$ so that (by (6)) $\alpha_{1}+\alpha_{2}=1$. Assume that both $\alpha_{1}>0$ and $\alpha_{2}>0$. We will show that this is impossible if $n>1$. Indeed, then $U_{i}=\left(\alpha_{i}\right)^{-1} u_{i}(i=1,2)$ is an isometry on $\ell_{2}^{n}$, such that, for any $e$ in the unit sphere of $E$, we have $e=\alpha_{1} U_{1}(e)+\alpha_{2} U_{2}(e)$. By the strict convexity of $\ell_{2}^{n}$, this implies that $U_{1}(e)=U_{2}(e)=e$ for all $e$. Moreover, by (7) we have $\gamma_{R}\left(U_{1}\right)=1$ and $\gamma_{C}\left(U_{2}\right)=1$. This implies that $E=R_{n}$ and $E=C_{n}$ completely isometrically, which is absurd when $n>1$. Hence, if $n>1$, we conclude that either $\alpha_{1}=0$ or $\alpha_{2}=0$, which implies either $\gamma_{C}\left(I d_{E}\right)=1$ or $\gamma_{R}\left(I d_{E}\right)=1$, equivalently either $E=C_{n}$ or $E=R_{n}$ completely isometrically. The remaining case $n=1$ is trivial.

Remark. Alternative proof: if $\mu(E)=1$, then, using (5) and the relexivity of $E$, we see that both for $E$ and $E^{* *}$ the identity factors through $\mathcal{K}^{* *}$, therefore $E$ is an injective operator space as well as its dual. Now, in [33], Ruan gives the complete list of the injective operator subspaces of finite dimensional $C^{*}$-algebras (see also [31] for more on this theme). Running down this list, and using an unpublished result of R. Smith saying that a finite dimensional injective operator space is completely contractively complemented in a finite dimensional $C^{*}$-algebra (see [2]), we find that $R_{n}$ and $C_{n}$ are the only possibilities.

Remark. We suspected that there did not exist an operator space $X$ such that (with the notation of Corollary 9) we had for any $E_{1}, E_{2}$ and any $u \in E_{1}^{*} \otimes E_{2}$

$$
\gamma_{X}(\tilde{u})=\|u\|_{\mu}
$$

and indeed C. Le Merdy has kindly provided us with an argument, as follows. Let $X$ be such a space. Let $E$ be an arbitrary finite dimensional subspace of $X$ and let $v_{E} \in E^{*} \otimes X$ denote the tensor representing the inclusion map $\tilde{v}_{E}: E \rightarrow X$. Then, by (3) and (8), $\gamma_{2}\left(\tilde{v}_{E}\right) \leq\left\|v_{E}\right\|_{\mu}=\gamma_{X}\left(\tilde{v}_{E}\right)=1$. By a well known ultraproduct argument (c.f. e.g. [27, p. 22]), this implies that $X$ is isometric to a Hilbert space. But then, a variant of the proof of Corollary 10 shows that we must have either $X=R$ or $X=C$ completely isometrically, and this is absurd.

However, (8) is true up to equivalence if we take for $X$ the direct sum of $R$ and $C$, in any reasonable way. For instance, it is easy to check that for any $u \in E_{1}^{*} \otimes E_{2}$ $\left(\tilde{u}: E_{1} \rightarrow E_{2}\right.$ being the associated finite rank operator) we have

$$
\frac{1}{2}\|u\|_{\mu} \leq \gamma_{R \oplus_{1}} c(\tilde{u}) \leq\|u\|_{\mu}
$$


Remark. It follows from Theorem 1 and the projectivity of Haagerup tensor product that $\otimes_{\mu}$ is also projective, i.e., if $q_{i}: F_{i} \rightarrow E_{i}(i=1,2)$ are quotient maps, so is $q_{1} \otimes q_{2}: F_{1} \otimes_{\mu} F_{2} \rightarrow E_{1} \otimes_{\mu} E_{2}$. On the other hand, $\otimes_{\mu}$ is not injective. To show this, consider the identity operator $i_{n}: R_{n} \cap C_{n} \rightarrow R_{n} \cap C_{n}$ and the natural (completely isometric) embedding $j_{n}: R_{n} \cap C_{n} \hookrightarrow R_{n} \oplus_{\infty} C_{n}$. The preceding remark (applied with $\left.\bar{u}=\left(j_{n} i_{n}\right)^{*}\right)$ implies that

$$
\left\|j_{n} i_{n}\right\|_{\left(R_{n}+C_{n}\right) \otimes_{\mu}\left(R_{n} \oplus_{\infty} C_{n}\right)} \leq 2
$$

However, by $\left[18\right.$, p. 912] we have $\gamma_{\mathcal{K}}\left(i_{n}\right) \geq(1+\sqrt{n}) / 2$, hence by Corollary 9 , we have

$$
\left\|i_{n}\right\|_{\mu} \geq(1+\sqrt{n}) / 2
$$

This proves that the tensor product $\otimes_{\mu}$ is not injective.

Remark. The examples in [27, Chapter 10] imply that there are (infinite dimensional) operator spaces $E$ such that $E^{*} \otimes_{\min } E=E^{*} \otimes_{\mu} E$ with equivalent norms, but $E$ is not completely isomorphic to $R$ or $C$, and actually (as a Banach space) $E$ is not isomorphic to any Hilbert space. Thus (in the isomorphic case) the second part of Corollary 10 does not seem to extend to the infinite dimensional setting without assuming some kind of approximation property.

We recall that any Hilbert space $H$ (resp. $K$ ) can be equipped with a column (resp. row) operator space, by identifying $H$ (resp. $K$ ) with $H_{c}=B(\mathrm{C}, H)$ (resp. with $K_{r}=B\left(K^{*}, C\right)$ ). Any operator space of this form will be called a "column space" (resp. a "row space").

We will use the following result from [22].

Theorem 11. ([22]) Let $E$ be an operator space such that $I d_{E}$ can be factorized completely boundedly through the direct sum $X=H_{c} \oplus_{1} K_{r}$ of a column space and a row space, (i.e., there are c.b. maps $u: E \rightarrow X$ and $v: X \rightarrow E$ such that $I d_{E}-v u$ ), then there are subspaces $E_{1} \subset H_{c}$ and $E_{2} \subset K_{r}$ such that $E$ is completely isomorphic to $E_{1} \oplus_{1} E_{2}$. More precisely, if we have $\|u\|_{c b}\|v\|_{c b} \leq c$ for some number $c$, then we can find a complete isomorphism $T: E \rightarrow E_{1} \oplus_{1} E_{2}$ such that $\|T\|_{c b}\left\|T^{-1}\right\|_{c b} \leq f(c)$ where $f: \mathbf{R}_{+} \rightarrow \mathbf{R}_{+}$is a certain function $f$.

Theorem 12. The following properties of an operator space E are equivalent:

(i) For any operator space $F$, we have $F \otimes_{\min } E=F \otimes_{\mu} E$ isomorphically.

(ii) $E$ is completely isomorphic to the direct sum of a row space and a column space.

Proof. The implication (i) $\Rightarrow$ (ii) is easy and left to the reader. Conversely, assume (i). Then, a routine argument shows that there is a constant $K$ such that for all $F$ and all $u$ in $F \otimes E$ we have $\|u\|_{\mu} \leq K\|u\|_{\min }$. Let $S \subset E$ be an arbitrary finite 
dimensional subspace let $j_{S}: S \rightarrow E$ be the inclusion map, and let $\hat{j}_{S} \in S^{*} \otimes E$ be the associated tensor. Then we have by (9) $\sup _{s} \gamma_{R \Theta_{1} c}\left(j_{s}\right)=\sup _{s}\left\|\hat{j}_{s}\right\|_{\mu} \leq K$. By a routine ultraproduct argument, this implies that the identity of $E$ can be written as in Theorem 11 with $c=K$, thus we conclude that $E \simeq E_{1} \oplus_{1} E_{2}$ where $E_{1}$ is a row space and $E_{2}$ a column space. Note that we obtain an isomorphism $T: E \rightarrow E_{1} \oplus_{1} E_{2}$ such that $\|T\|_{c b}\left\|T^{-1}\right\|_{c b} \leq f(K)$. In particular, if $E$ is finite dimensional, we find $T$ such that

$$
\|T\|_{c b}\left\|T^{-1}\right\|_{c b} \leq f\left(\left\|i_{E}\right\|_{\mu}\right) .
$$

We now turn to a result at the root of the present investigation. Let $E$ be an operator space and let $A$ be a unital operator algebra. for any $x$ in $E \otimes A$, we define

$$
\delta(x)=\sup \|\sigma \cdot \pi(x)\|
$$

where the supremum runs over all pairs $(\sigma, \pi)$ where $\sigma: E \rightarrow B(H)$ is a complete contraction, $\pi: A \rightarrow B(H)$ a morphism and moreover $\sigma$ and $\pi$ have commuting ranges.

Let $E \otimes_{\delta} A$ be the completion of $E \otimes A$ for this norm. We may clearly also view $E \otimes_{\delta} A$ as an operator space using the embedding $x \rightarrow \oplus_{(\sigma, \pi)}(\sigma \cdot \pi)(x)$ where the direct sum runs over all pairs as above. Note that the natural inclusion $E \otimes_{\delta} A \subset O A(E) \otimes_{\max } A$ is a complete isometry. In particular, if $F$ is another operator space, we have a natural completely isometric embedding of $E \otimes_{\mu} F$ into $E \otimes_{\delta} O A(F)$, which explains the connection of the delta tensor product to the present paper. Then we may state.

Theorem 13. Consider the linear mapping $q: A \otimes E \otimes A \rightarrow E \otimes A$ defined by

$$
q(a \otimes e \otimes b)=e \otimes(a b) .
$$

This mapping $q$ defines a complete metric surjection from $A \otimes_{h} E \otimes_{h} A$ onto $E \otimes_{\delta} A$. More precisely, for any $n$ and any $x$ in $M_{n}(E \otimes A)$ with $\|x\|_{M_{n}\left(E \otimes_{b}\right)}<1$, there is $\tilde{x}$ in $M_{n}(A \otimes E \otimes A)$ with $\|\tilde{x}\|_{M_{n}\left(A \otimes_{h} E \otimes_{h} A\right)}<1$ such that $I_{M_{n}} \otimes q(\tilde{x})=x$.

Remark. This statement is due to the second author [30] (who is indebted to C. Le Merdy for observing this useful reformulation). A proof (somewhat different from the original one in [36]) can be given following the lines of the above proof of Theorem 1 (here, one considers $O A(E) \otimes_{\max } A$ as a quotient of the free product $O A(E) * A$, and one uses the fact that $A$.E.A. spans inside $O A(E) * A$ a subspace completely isometric to $A \otimes_{h} E \otimes_{h} A$ ), so we skip it. This result yields simpler proofs and extensions of several statements concerning nuclear $C^{*}$-algebras. See the final version of [30] for more details on this topic. 


\section{An alternate approach}

Using $[9,10,25]$, one can see that the following statement is a dual reformulation of Theorem 1.

Theorem 18. Let $E_{1}, E_{2}$ be two operator spaces, and let $\varphi: E_{1} \otimes E_{2} \rightarrow B(\mathcal{H})$ be a linear mapping. The following are equivalent:

(i) $\|\varphi\|_{c b\left(E_{1} \otimes_{\mu} E_{2}, B(\gamma)\right)} \leq 1$.

(ii) For some Hilbert space $H$, there are complete contractions $\alpha_{1}: E_{1} \rightarrow B(H, \mathcal{H})$, $\alpha_{2}: E_{2} \rightarrow B(\mathcal{H}, H)$, and $\beta_{1}: E_{1} \rightarrow B(\mathcal{H}, H), \beta_{2}: E_{2} \rightarrow B(H, \mathcal{H})$, such that

$$
\forall\left(x_{1}, x_{2}\right) \in E_{1} \times E_{2} \quad \varphi\left(x_{1} \otimes x_{2}\right)=\alpha_{1}\left(x_{1}\right) \alpha_{2}\left(x_{2}\right)=\beta_{2}\left(x_{2}\right) \beta_{1}\left(x_{1}\right) .
$$

(iii) For some Hilbert space $H$, there are complete contractions $\sigma_{i}: E_{i} \rightarrow B(H)$ $(i=1,2)$, with commuting ranges, and contractions $V: \mathcal{H} \rightarrow H$ and $W: H \rightarrow \mathcal{H}$, such that

$$
\forall\left(x_{1}, x_{2}\right) \in E_{1} \times E_{2} \quad \varphi\left(x_{1} \otimes x_{2}\right)=W \sigma_{1}\left(x_{1}\right) \sigma_{2}\left(x_{2}\right) V
$$

Proof. Assume (1). By Remark 2, $\varphi$ defines a complete contraction into $B(\mathcal{H})$ both from $E_{1} \otimes_{h} E_{2}$ and from $E_{2} \otimes_{h} E_{1}$. Then (ii) follows from the Christensen-Sinclair factorization theorem for bilinear maps, extended to general operator spaces by Paulsen and Smith in [25]. Now assume (ii). Let $H_{1}=\mathcal{H}, H_{2}=H$ and $H_{3}=\mathcal{H}$. We define maps $\sigma_{1}: E_{1} \mapsto B\left(H_{1} \oplus H_{2} \oplus H_{3}\right)$ and $\sigma_{2}: E_{2} \rightarrow B\left(H_{1} \oplus H_{2} \oplus H_{3}\right)$ using matrix notation, as follows

$$
\begin{aligned}
& \sigma_{1}\left(x_{1}\right)=\left(\begin{array}{ccc}
0 & \alpha_{1}\left(x_{1}\right) & 0 \\
0 & 0 & \beta_{1}\left(x_{1}\right) \\
0 & 0 & 0
\end{array}\right) \\
& \sigma_{2}\left(x_{2}\right)=\left(\begin{array}{ccc}
0 & \beta_{2}\left(x_{2}\right) & 0 \\
0 & 0 & \alpha_{2}\left(x_{2}\right) \\
0 & 0 & 0
\end{array}\right) .
\end{aligned}
$$

Then, by (ii) we have

$$
\sigma_{1}\left(x_{1}\right) \sigma_{2}\left(x_{2}\right)=\sigma_{2}\left(x_{2}\right) \sigma_{1}\left(x_{1}\right)=\left(\begin{array}{ccc}
0 & 0 & \varphi\left(x_{1} \otimes x_{2}\right) \\
0 & 0 & 0 \\
0 & 0 & 0
\end{array}\right)
$$


hence $\sigma_{1}, \sigma_{2}$ have commuting ranges, and are complete contractions. Therefore if we let $W: H_{1} \oplus H_{2} \oplus H_{3} \rightarrow \mathcal{H}$ be the projection onto the first coordinate and $V: \mathcal{H} \rightarrow H_{1} \oplus H_{2} \oplus H_{3}$ be the isometric inclusion into the third coordinate, then we obtain (iii). Finally, the implication (iii) implies (i) is obvious by the very definition of $E_{1} \otimes_{\mu} E_{2}$.

Alternate proof of Theorem $1(n=2)$. By duality, it clearly suffices to show that for any linear map $\varphi: E_{1} \otimes E_{2} \rightarrow B(\mathcal{H})$ the norms $\|\varphi\|_{c b\left(E_{1} \otimes_{\mu} E_{2} \rightarrow B(\mathcal{H})\right)}$ and $\|\varphi Q\|_{c b}$ are equal. But this is precisely the meaning of the equivalence between (i) and (ii) in Theorem 18. Thus we conclude that Theorem 18 implies Theorem 1 for $n=2$.

In the $n$-linear case with $n>2$, the preceding argument yields the following statement, which seems rather different from the $n$-linear version of Theorem 1 . To formulate this we need to introduce a variant of the definitions appearing in (1) and (2) where we restrict ourselves to the $n$ cyclic permutations of $\{1, \ldots, n\}$. We will say that an $n$-tuple of operators $\left(T_{1}, \ldots, T_{n}\right)$ on $H$ cyclically commute if we have $T_{1} \ldots T_{n}=T_{\sigma(1)} \ldots T_{\sigma(n)}$ for any cyclic permutation $\sigma$. (When $n=2$, this is the same as ordinary commutation.) Let $E_{1}, \ldots, E_{n}$ be operator spaces. We will define the cyclic analogue of the $\mu$-tensor product. We first define the norm as follows.

$$
\forall x \in E_{1} \otimes \cdots \otimes E_{n} \quad\|x\|_{c}=\sup \left\|\sigma_{1} \cdot \ldots \cdot \sigma_{n}(x)\right\|_{B(H)}
$$

where the supremum runs over all possible $H$ and all $n$-tuples $\left(\sigma_{i}\right)$ of complete contractions $\sigma_{i}: E_{i} \rightarrow B(H)$ which cyclically commute (i.e., such that $\left(T_{1}, \ldots, T_{n}\right)$ cyclically commute for any choice of $T_{i}$ in the range of $\sigma_{i}$ ). We will denote by $\left(E_{1} \otimes E_{2} \cdots \otimes E_{n}\right)_{c}$ the completion of $E_{1} \otimes \cdots \otimes E_{n}$ for this norm, and will consider it as an operator space in the same way as in (2).

Theorem 19. Let $E_{1}, \ldots, E_{n}$ be an n-tuple of operator spaces. Let $Y_{1}=E_{1} \otimes_{k}$ $E_{2} \ldots \otimes_{h} E_{n}$ and, for $k \geq 2, \quad Y_{k}=E_{k} \otimes_{h} E_{k+1} \ldots \otimes_{h} E_{n} \otimes_{h} E_{1} \otimes_{h} \ldots \otimes_{h} E_{k-1}$. Let $L_{c}=$ $Y_{1} \oplus_{1} \ldots \oplus_{1} Y_{n}$. Let $Q_{c}$ be the natural completely contractive mapping from $L_{c}$ to $\left(E_{1} \otimes \cdots \otimes E_{n}\right)_{c}$, and let $\Phi_{c}: L_{c} / \operatorname{ker}\left(Q_{c}\right) \rightarrow\left(E_{1} \otimes \cdots \otimes E_{n}\right)_{c}$ be the canonically associated map. Then $\Phi_{c}$ is a complete isometry.

We leave the proof, which is an easy modification of the argument for Theorem 18 , to the reader. Note that we do not see how to prove Theorem 19 using the ideas of Section 1 (nor do we see how to prove Theorem 1 when $n>2$ using the ideas of Section 2).

Remark 20. Following [11], we say that a collection of Banach algebras which is stable by arbitrary direct sums, subalgebras and quotients is a variety. Let $\mathcal{V}$ be a variety formed of unital operator algebras. Of course, we are interested in their operator space (and not only their Banach space) structure and we use unital completely contractive homomorphisms as morphisms. One of the advantages of the 
first proof over the second one is that its "categorical principle" can be easily adapted to compute the analogue of the $\mu$-tensor product obtained when one restricts all maps to take their values into an algebra belonging to some fixed given variety $\mathcal{V}$.

Acknowledgement. We are very grateful to $\mathrm{C}$. Le Merdy for letting us include several useful remarks and his example showing the non-associativity of the $\mu$-tensor product.

\section{REFERENCES}

1. D. BLECHER, Tensor products of operator spaces II, Canadian J. Math. 44 (1992), 75-90.

2. D. BLecher, The standard dual of an operator space, Pacific J. Math. 153 (1992), 15-30.

3. D. BLECHER, A completely bounded characterization of operator algebras, Math. Ann. 303 (1995), 227-239.

4. D. Blecher and V. Paulsen, Tensor products of operator spaces, J. Funct. Anal. 99 (1991), 262-292.

5. D. BLeCher and V. Paulsen, Explicit constructions of universal operator algebras and applications to polynomial factorization, Proc. Amer. Math. Soc. 112 (1991), 839-850.

6. D. Blecher, Z. J. RuAN and A. Sinclair, A characterization of operator algebras, $J$. Funct. Anal. 89 (1990), 188-201.

7. D. BLeCher and R. SMith, The dual of the Haagerup tensor product, J. London Math. Soc. 45 (1992), 126-144.

8. E. Christensen, E. Effros and A. Sinclair, Completely bounded multilinear maps and $C$-algebraic cohomology, Invent. Math. 90 (1987), 279-296.

9. E. Christensen and A. Sinclair, Representations of completely bounded multilinear operators, J. Funct. Anal. 72 (1987), 151-181.

10. E. Christensen and A. Sinclair, A survey of completely bounded operators, Bull. London Math. Soc. 21 (1989), 417-448.

11. P. Dixon, Varieties of Banach algebras, Quart. J. Math. Oxford 27 (1976), 481-487.

12. E. Erfros and Z. J. RUAN, On approximation properties for operator spaces, Internat. J. Math. 1 (1990), 163-187.

13. E. EFrRos and Z. J. RUAN, A new approach to operator spaces, Canadian Math. Bull. 34 (1991), 329-337.

14. E. Erfros and Z. J. RUAN, Self duality for the Haagerup tensor product and Hilbert space factorization, J. Funct. Anal. 100 (1991), 257-284.

15. E. EfrRos and Z. J. RuAN, On the abstract characterization of operator spaces, Proc. Amer. Math. Soc. 119 (1993), 579-584.

16. E. EFrros and Z. J. RUAN, Mapping spaces and liftings for operator spaces, Proc. London Math. Soc. 69 (1994), 171-197.

17. E. EFrRos and Z. J. RUAN, The Grothendieck-Pietsch and Dvoretzky-Rogers Theorems for operator spaces, J. Funct. Anal. 122 (1994), 428-450.

18. U. HaAgeruP and G. Pisier, Bounded linear operators between $C^{*}$-algebras, Duke Math. J. 71 (1993), 889-925. 
19. M. JUnge and G. Pisier, Bilinear forms on exact operator spaces and $B(H) \otimes B(H)$, Geom. Funct. Anal. 5 (1995), 329-363.

20. R. KADISON and J. RINGROSE, Fundamentals of the theory of operator algebras, II (Academic Press, New York, 1986).

21. E. KirchberG, On non-semisplit extensions, tensor products and exactness of group $C^{*}$. algebras, Invent. Math. 112 (1993), 449-489.

22. T. OIKHBERG, Direct sums of homogeneous operator spaces, article in preparation.

23. V. Paulsen, Completely bounded maps and dilations (Pitman Research Notes in Math. 146, Longman, Wiley, New York, 1986).

24. V. PAULSEN and S. Power, Tensor products of non-self adjoint operator algebras, Rocky Mountain J. Math. 20 (1990), 331-350.

25. V. Paulsen and R. Smith, Multilinear maps and tensor norms on operator systems, $J$. Funct. Anal. 73 (1987), 258-276.

26. V. Pestov, Operator spaces and residually finite-dimensional $C^{*}$-algebras, J. Funct. Anal. 123 (1994), 308-317.

27. G. PISIER, Factorization of linear operators and the Geometry of Banach spaces (CBMS (Regional conferences of the A.M.S.) 60, 1986), Reprinted with corrections 1987.

28. G. PISIER, The operator Hilbert space $O H$, complex interpolation and tensor norms, Mem. Amer. Math. Soc. 122585 (1996), 1-103.

29. G. Pisier, A simple proof of a theorem of Kirchberg and related results on $C^{*}$-norms, $J$. Operator Theory 35 (1996), 317-335.

30. G. Pisier, An introduction to the theory of operator spaces, preprint, to appear.

31. A. G. Robertson, Injective matricial Hilbert spaces, Math. Proc. Cambridge Philos. Soc. 110 (1991), 183-190.

32. Z. J. Ruan, Subspaces of $C^{*}$-algebras, J. Funct. Anal. 76 (1988), 217-230.

33. Z. J. RUAN, Injectivity and operator spaces, Trans. Amer. Math. Soc. 315 (1989), 89-104.

34. M. TAKESAKI, Theory of Operator Algebras I (Springer-Verlag, New York, 1979).

The UNIVERsity of TeXas

AUSTIN

TX 78712

U.S.A.

\section{TeXas A\&M UNIVERSITY \\ College Station \\ TX 77843 \\ U.S.A.}

and

UNiversité Paris 6

PARIS

France 